\title{
Label-free optical control of arterial contraction
}

\author{
Chulhee Choi \\ Korea Advanced Institute of Science and Technology \\ (KAIST) \\ Department of Bio and Brain Engineering \\ Cell Signaling and Bioimaging Laboratory \\ and \\ Graduate School of Medical Science and Engineering \\ and \\ KAIST Institute for BioCentury \\ 335 Gwahak-ro, Yuseong-gu \\ Daejeon 305-701, Korea
}

\author{
Myunghwan Choi \\ Jonghee Yoon \\ Korea Advanced Institute of Science and Technology \\ (KAIST) \\ Department of Bio and Brain Engineering \\ Cell Signaling and Bioimaging Laboratory \\ 335 Gwahak-ro, Yuseong-gu \\ Daejeon 305-701, Korea
}

\begin{abstract}
The diameters of blood vessels, especially in the brain, change dynamically over time to provide sufficient blood supply as needed. No existing technique allows noninvasive control of vascular diameter in vivo. We report that label-free irradiation with a femtosecond pulsed laser can trigger blood vessel contraction in vivo. In response to laser irradiation, cultured vascular smooth muscle cells showed a rapid increase in calcium concentration, followed by cell contraction. In a murine thinned skull window model, laser irradiation focused in the arterial vessel wall caused localized vascular contraction, followed by recovery. The nonlinear nature of the pulsed laser allowed highly specific targeting of subcortical vessels without affecting the surrounding region. We believe that femtosecond pulsed laser irradiation will become a useful experimental tool in the field of vascular biology. () 2010 Society of Photo-Optical Instrumentation Engineers. [DOI: 10.1117/1.3316404]
\end{abstract}

Keywords: femtosecond laser; vascular smooth muscle; label free; noninvasive.

Paper 09278R received Jul. 1, 2009; revised manuscript received Nov. 8, 2009; accepted for publication Dec. 28, 2009; published online Feb. 23, 2010.

\section{Introduction}

Tissue demands for the nutrients and oxygen delivered by blood change according to cellular activity. To meet these needs with a limited blood supply, the vascular system dynamically regulates regional blood flow, and the control of arterial tone is integral to regulating tissue perfusion by blood. ${ }^{1}$ Arterial tone is determined by the contraction or dilation of vascular smooth muscle in the tunica media layer of the arterial wall, and the intracellular calcium dynamics in vascular smooth muscle cells (VSMCs) are a key determinant of tone. ${ }^{2}$

The central nervous system, especially the brain, exhibits tight coupling between neuronal metabolic activity and vascular tone. ${ }^{3}$ This neurovascular coupling is used to indirectly measure neural activity in functional magnetic resonance imaging (fMRI), and its malfunctioning is related to neurodegenerative diseases and stroke. ${ }^{4}$ However, the study of neurovascular coupling has been hampered by the lack of appropriate experimental tools to regulate arterial tone in vivo. Existing methods of controlling vessel tone, including direct electrical stimulation and treatment with vasoactive agents, have low spatial specificity and a high degree of invasiveness. $^{5}$

Near-infrared (NIR) femtosecond pulsed lasers have been widely used for bioimaging because of their deep penetration, reduced scattering, and localized nonlinear absorption. ${ }^{6}$ These advantages have enabled the optical modulation of live cells and tissues in live animals - for example, the generation of intracellular calcium, ${ }^{7}$ dissection of intracellular organelles, ${ }^{8}$ gene transfection, ${ }^{9}$ induction of neuronal firing, ${ }^{10}$ and disruption of blood flow. ${ }^{11}$ Especially, as the generation of calcium

Address all correspondence to: Chulhee Choi, KAIST, Department of Bio and Brain Engineering, Cell Signaling and Bioimaging Laboratory, 373-1 Guseongdong, Yuseong-gu, Daejeon 305-701, Korea. Tel: 82-42-350-4321; Fax: 82-42 350-4380. E-mail: cchoi@kaist.ac.kr in cardiomyocytes elicits cell contraction, timely irradiation has been proposed as a cardiac pacemaker. ${ }^{12}$ Here, we studied the effects of a femtosecond laser on vascular smooth muscle in vitro and in vivo.

\section{Materials and Methods}

\subsection{Cell Culture}

Primary cultures of rat aortic smooth muscle cells were prepared from the thoracic and abdominal aortas of 5-week-old male Sprague-Dawley rats (150 to $200 \mathrm{~g}$ ), as described previously. ${ }^{13}$ The cells were maintained in Dulbecco's modified Eagle's medium (DMEM) supplemented with $10 \%$ fetal bovine serum and were used between passages 5 and 10 . Purity $(>95 \%)$ was confirmed by the staining of $\alpha$-smooth muscle actin.

\subsection{Murine Thinned Skull Model}

All experiments used 7- to 10-week-old male ICR mice (Charles River Japan, Yokohama, Japan). The procedures for the thinned skull preparation were as described previously. ${ }^{14}$ After anesthesia with an intraperitoneal injection of ketaminexylazine, the scalp is removed and the skull is glued to a custom-designed metal plate (Namil Optical Components, Incheon, Korea) using dental cement. Using a high-speed microdrill, a circular area of skull, 2 to $4 \mathrm{~mm}$ in diameter, over the region of interest is thinned under a dissection microscope until a thin, smooth preparation is achieved. The animal care and experimental procedures were performed under the approval of the Animal Care Committee of KAIST (Daejeon, Korea).

$1083-3668 / 2010 / 15(1) / 015006 / 6 / \$ 25.00$ ○ 2010 SPIE 
a

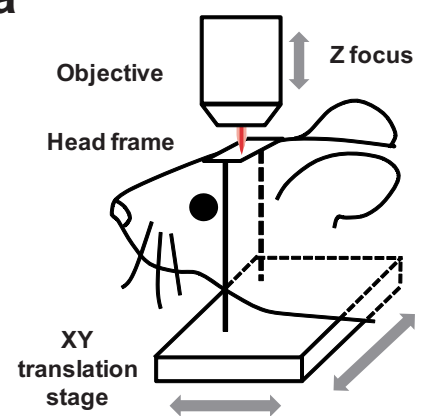

b

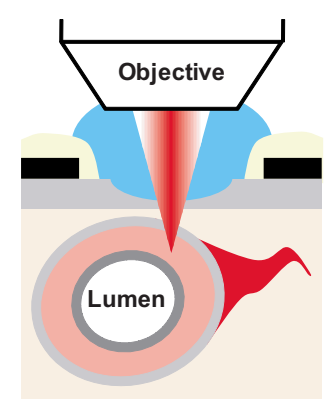

C

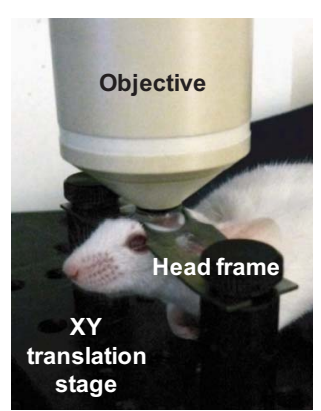

Fig. 1 In vivo experimental setup. (a, b) Schematics of the animal imaging setup. aCSF, artificial cerebrospinal fluid. (c) A photograph of the animal imaging setup.

\subsection{Two-Photon Fluorescence Imaging}

We used two-photon laser scanning microscopy (LSM510; Carl Zeiss, Oberkochen, Germany) with a femtosecond pulsed laser (Chameleon; Coherent, Santa Clara, California) tuned to $800 \mathrm{~nm}$ and a water immersion objective lens $(20 \times, 1.0 \mathrm{nu}-$ merical aperture). For cellular calcium imaging, cultured cells were loaded with $10 \mu \mathrm{mol} / \mathrm{L}$ of the acetoxymethyl form of Fluo-4 (Fluo-4AM; Molecular Probes, Eugene, Oregon) at $37{ }^{\circ} \mathrm{C}$ for $40 \mathrm{~min}$ in DMEM and then washed with phosphate-buffered saline (PBS). For membrane staining, the cells were loaded with $5 \mu \mathrm{g} / \mathrm{ml}$ Alexa Fluor 594-conjugated wheat germ agglutinin (WGA-Alexa Fluor 594; Invitrogen, Carlsbad, California) at $37{ }^{\circ} \mathrm{C}$ for 5 min in Hank's balanced salt solution and then washed twice with PBS. For animal imaging, after the skull thinning surgery, we intravenously injected 2-MDa FITC-dextran (100 $\mu \mathrm{l}$ of $2 \% \mathrm{w} / \mathrm{v}$ in PBS; FD-2000S; Sigma), and the mice were placed on a customized frame (Namil Optical Components, Incheon, Korea) designed for minimizing movement artifacts (Fig. 1). We imaged the baseline state of a region of interest for $10 \mathrm{~s}$ and subsequently imaged the region for up to 1 to 2 min after laser irradiation. The average laser power used for imaging was less than $50 \mathrm{~mW}$.

\subsection{Laser Irradiation}

The optical path and laser source for optical intervention were the same as those used in the two-photon fluorescence imaging. For the in vitro study, we selected a target region of $<0.09 \mu \mathrm{m}^{2}$ in the cytosol of a cultured VSMC. The irradiation duration was fixed at $1.6 \mathrm{~ms}$, and the average laser power was set to 300 to $450 \mathrm{~mW}$. We started with low laser power and increased it until a calcium response was observed. For the animal experiments, we selected target arteries that coursed parallel to the cortical surface and that had lumen diameters of 15 to $30 \mu \mathrm{m}$ and subsurface depths of up to $100 \mu \mathrm{m}$ in a thinned skull window model. The arterial vasculature was confirmed by measuring the direction of blood flow in the branching vessels. To target the media layer of the arterial wall, a region located 3 to $5 \mu \mathrm{m}$ from the luminal surface was selected for irradiation. The irradiation duration was fixed at $1.6 \mathrm{~ms}$. The average laser power was set initially at $300 \mathrm{~mW}$ and was increased to $900 \mathrm{~mW}$, until vasoconstriction was observed.

\subsection{Data Analysis}

We used ImageJ or MATLAB for image processing and data quantification. Data are expressed as the means \pm S.E.M.

\section{Results}

\subsection{Laser-Induced Calcium Signals in VSMCs}

To test whether femtosecond pulsed laser stimulation can trigger calcium waves in VSMCs, we irradiated rat aortic VSMCs stained with the calcium indicator Fluo-4AM. A target cell was selected using the basal calcium fluorescence, and the cytosol region was irradiated as described in Sec. 2. Before starting the laser stimulation experiments, we confirmed that repetitive imaging with an average power of less than $50 \mathrm{~mW}$ did not cause any notable changes in the calcium signal or cell viability (data not shown). Considering that the laser-induced calcium response in other cell types is known to exhibit a power-dependent, all-or-nothing response, we gradually increased the laser power used for cell stimulation until we observed a change in the calcium signal in the cell. ${ }^{15}$ For most cells, we observed an approximately twofold increase in calcium fluorescence signal, which reached a maximum at $\sim 1 \mathrm{~s}$ and was followed by a subsequent decrease to the basal level. Nonresponding cells underwent necrotic cell death, characterized by loss of the calcium signal and membrane integrity, on repeated irradiation with increasing laser power. In the irradiated cell, increase in a calcium signal was observed in both nuclear and cytosol regions, with a greater signal intensity in the nuclear region, and punctate calcium pattern was observed in the cytosol at the later time points. The calcium wave propagated to the neighboring cells, and the propagation was blocked by treating with a gap junction blocker, 18 $\beta$-glycyrrhetinic acid, suggesting that the gap junction mediated propagation of the calcium signal (data not shown). To produce a dose-response curve for the laser-induced calcium signals, we irradiated randomly selected cells $(n=30$ for each laser power) with seven different laser powers $(32.9,99,164$, $230,296,370,444$, and $740 \mathrm{~mW}$ ) and found that $300 \mathrm{~mW}$ was enough to trigger a calcium signal in VSMCs (Fig. 2; also see Video 1). We used this laser power for the following in vitro experiments. 
a

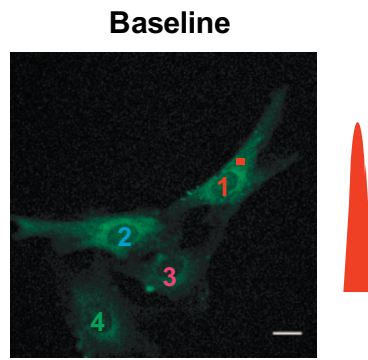

b

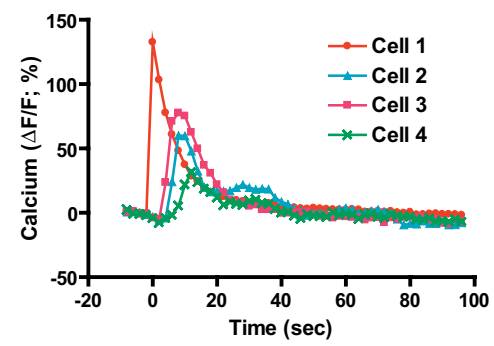

$2 s$

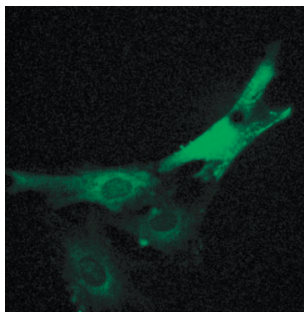

C

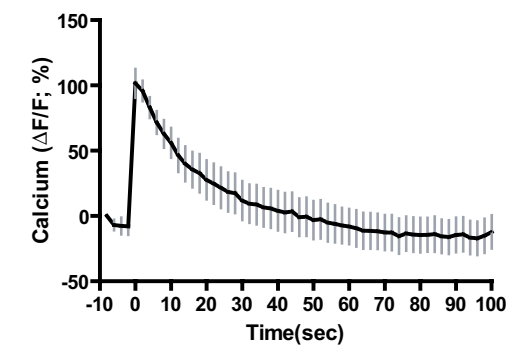

$30 \mathrm{~s}$

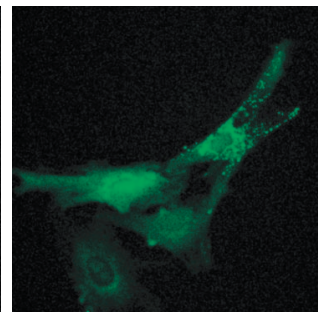

Time(sec)

d
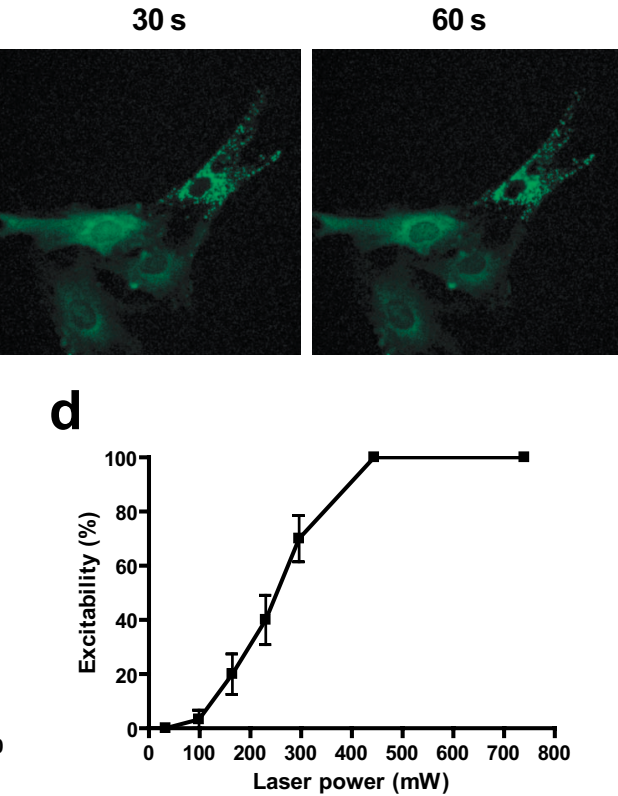

Fig. 2 Laser-induced calcium signal in a VSMC. (a) Representative temporal dynamics of the laser-induced calcium wave in a cultured VSMC. The red dot indicates the irradiated region. The numbers above each image indicate the time after laser stimulation at 0 s. Scale bar, $20 \mu m$. (b) Quantification of laser-induced calcium dynamics in the irradiated cell and the neighboring cells. The numbers in the baseline image in (a) indicate the corresponding cells. (c) Quantification of laser-induced calcium dynamics. The gray lines indicate error bars ( $n=15$ ). (d) Laser power dependency of calcium wave generation in VSMCs. $n=30$ for each point.

\subsection{Laser-Induced Contraction of VSMCS}

Elevation of intracellular calcium ion concentration is a wellknown signaling pathway responsible for VSMC contraction. ${ }^{1}$ We investigated whether the laser-induced calcium increase leads to VSMC contraction. To visualize cell contraction, we used a plasma membrane-staining dye, WGA-Alexa Fluor 594, together with a calcium indicator. Repetitive imaging did not show any noticeable changes in plasma membrane morphology, except minimal photobleaching acceptable for structural imaging (data not shown). Subthreshold laser stimulation

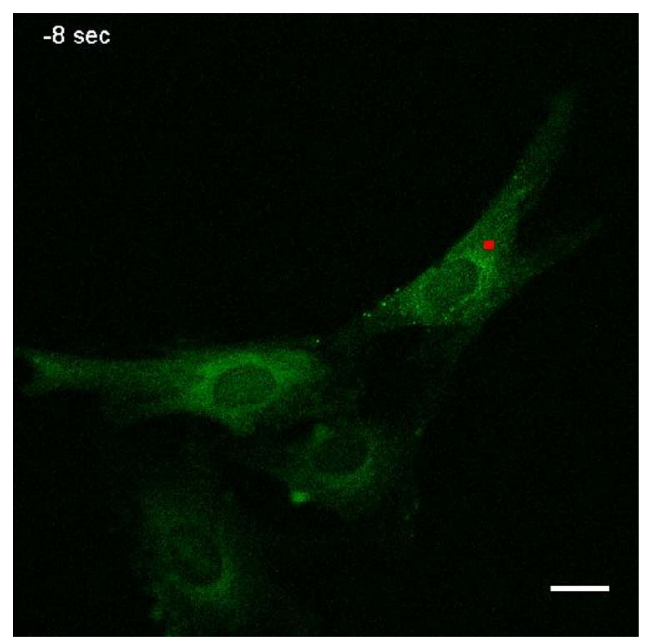

Video 1 Representative temporal dynamics of the laser-induced calcium wave in a cultured VSMC. The red dot indicates the irradiated region. Scale bar, $20 \mu \mathrm{m}$ (MPEG, 1.4 MB).

[URL: http://dx.doi.org/10.1117/1.3316404.1]. did not induce changes in cell morphology or the calcium signal. Following suprathreshold laser stimulation, the plasma membrane showed gradual shrinkage, indicating active cell contraction ( $2.6 \pm 0.2 \%$ cell area change per min), along with rapid calcium responses consistent with the previous experiments. After $100 \mathrm{~s}$, the cell area had changed to $97 \pm 0.3 \%$ $(n=15)$ of the baseline area (Fig. 3; also see Video 2).

\subsection{Laser-Induced Arterial Contraction}

Next, we examined whether this laser-induced VSMC contraction occurred in a live animal (Fig. 4). We used a murine thinned skull model to optically access the cortical vasculature in the brain, in a minimally invasive manner. The vascular lumen was visualized by an intravenous injection of 2-MDa FITC-dextran, which cannot pass through the vessel wall. Arteries were selected by measuring the blood flow directions in the branching vessels, using a line-scanning method. To target the arterial media layer containing smooth muscle, a region located 3 to $5 \mu \mathrm{m}$ from the luminal surface was irradiated.

Laser irradiation focused in the arterial vessel wall caused localized vascular contraction, followed by recovery. The vascular lumen contracted to $74.3 \pm 3.9 \%(n=5)$ of the baseline diameter, and contraction peaked at $\sim 10 \mathrm{~s}$ after irradiation. Recovery was completed within $1 \mathrm{~min}$, with almost complete reversal of the vascular diameter. The arteriolar contraction dynamics with laser stimulation were comparable to those with other stimuli. ${ }^{1}$ Irradiation of a spot in the vessel wall resulted in the rapid lateral spread of contraction within a few seconds. 
a Baseline
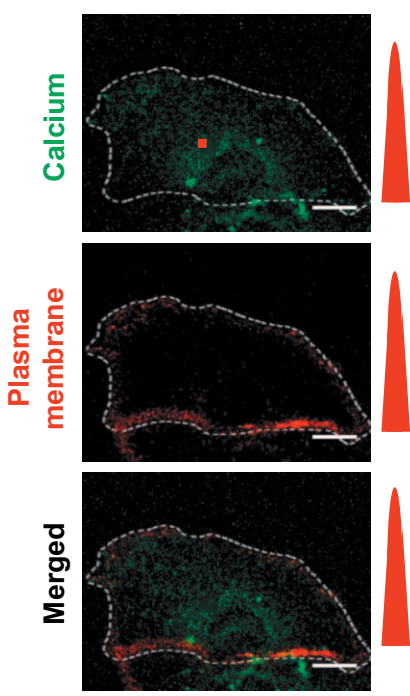

b

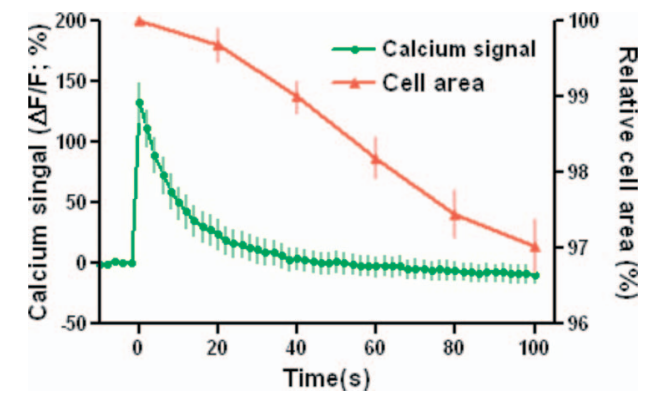

$10 \mathrm{~s}$
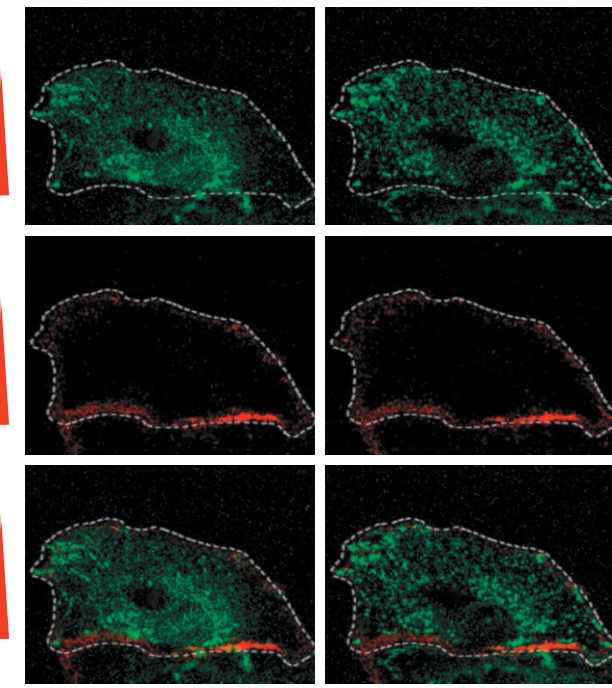

$100 \mathrm{~s}$
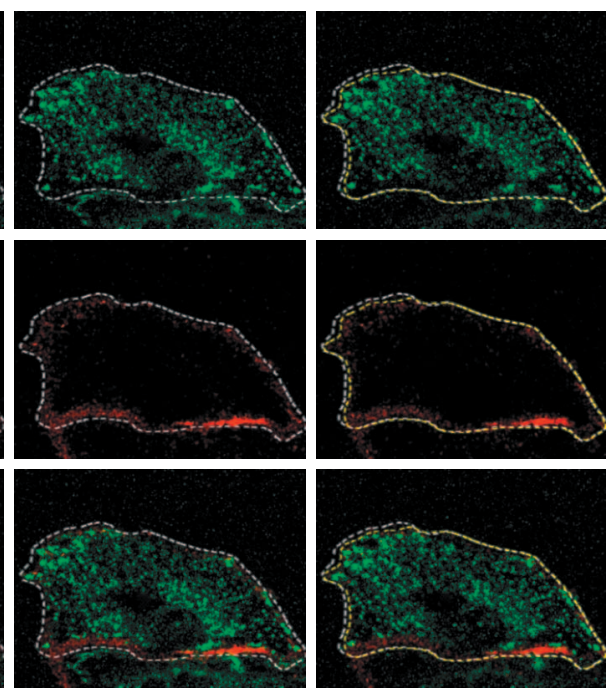

Fig. 3 Laser-induced VSMC contraction. (a) Representative temporal dynamics of laser-induced VSMC contraction. The calcium signal and membrane morphology were imaged simultaneously. The white dashed lines indicate the cell margin at the baseline, and the yellow dashed lines indicate the cell margin at $100 \mathrm{~s}$. The numbers indicate the time after laser stimulation at $0 \mathrm{~s}$. The red dot indicates the irradiated region. Scale bar, $20 \mu \mathrm{m}$. (b) Quantification of laser-induced calcium dynamics and cell area change $(n=15)$.

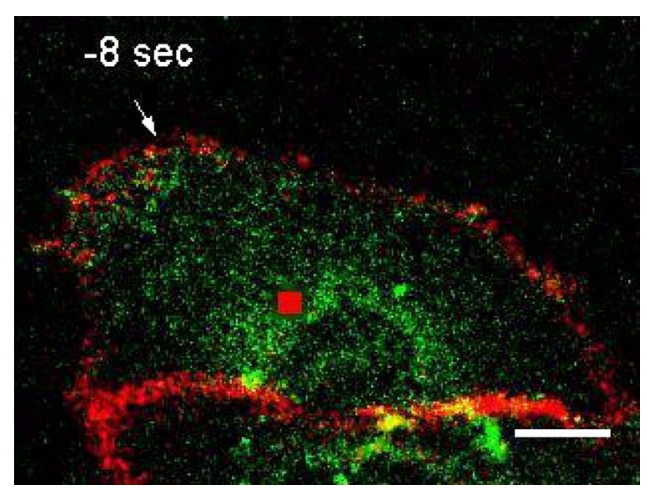

Video 2 Representative temporal dynamics of the laser-induced VSMC contraction. The red dot indicates the irradiated region. Scale bar, $20 \mu \mathrm{m}$ (MPEG, $1.4 \mathrm{MB})$.

[URL: http://dx.doi.org/10.1117/1.3316404.2].

\section{Discussion}

We demonstrated that a femtosecond pulsed laser can induce the contraction of arterial blood vessels without the use of exogenous probes such as caged molecules. To our knowledge, this is the first methodological report on noninvasive optical control of vascular tone in vivo. Using a femtosecond pulsed laser, we could precisely target the subsurface vasculature, without affecting the out-of-focus regions. This novel method will help to answer previously experimentally unapproachable questions in vascular physiology. As laser irradiation has only spatial specificity, without specific chemical targets, any subcellular structures in the focal volume are potential candidates responsible for the calcium response. A focused ultrashort pulsed laser can locally disrupt subcellular structures such as the plasma membrane, chromosomes, and mitochondria. ${ }^{8}$ Given that we observed a response in randomly selected regions in the cytosol, the target may be a ubiquitous cytosolic structure with a role in intracellular calcium homeostasis, possibly the plasma membrane or endoplasmic reticulum. Pulsed laser irradiation that is focused on the plasma membrane can induce the formation of a transient pore, leading to the influx of extracellular calcium. ${ }^{16}$ Disruption of the endoplasmic reticulum, the main intracellular cal- 
a

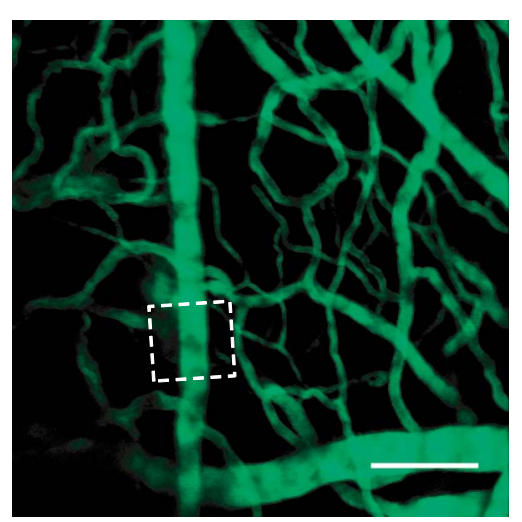

C

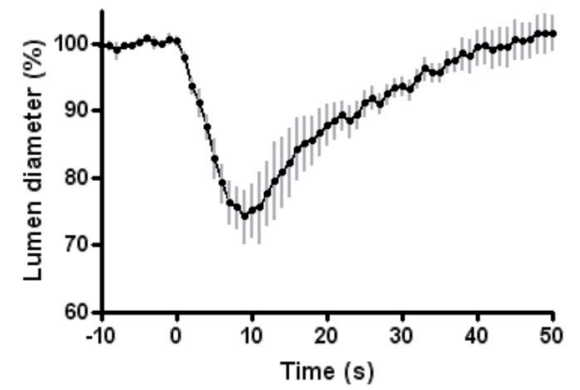

b

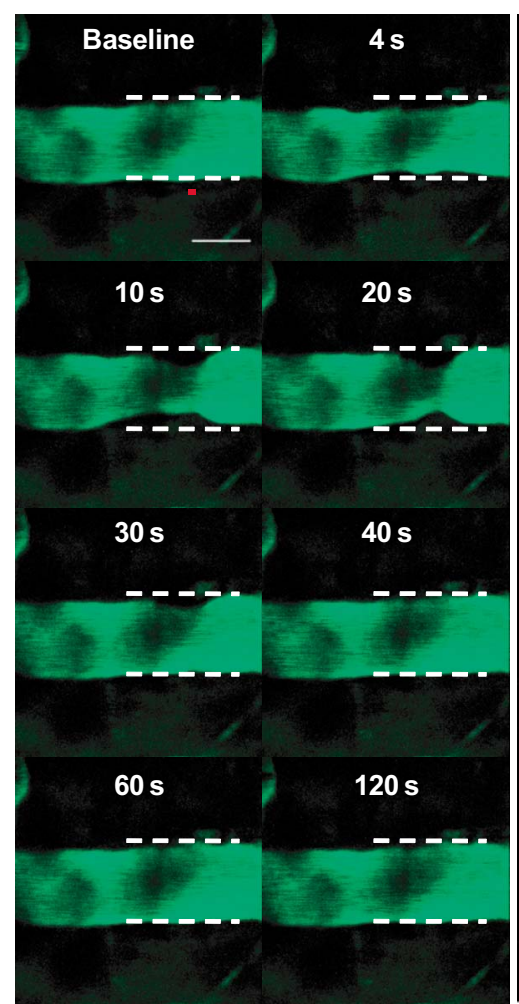

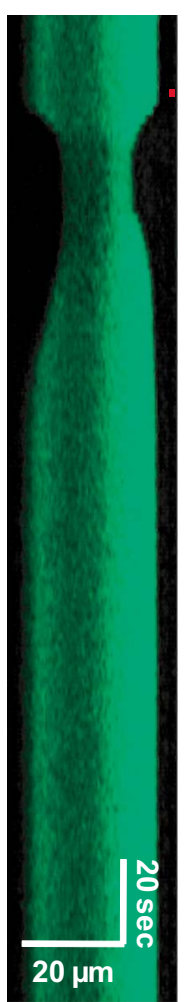

Fig. 4 Laser-induced cortical arterial constriction. (a) Vascular structure imaged through a thinned skull window after an intravenous injection of fluorescein conjugated to 2-MDa dextran. The dashed square indicates the region of interest for images in (b). Scale bar, $100 \mu \mathrm{m}$. (b) Representative time points after irradiation. The red dot and dashed line indicate the irradiated region and baseline vessel wall, respectively. Scale bar, $20 \mu \mathrm{m}$. A line scan image reconstructed using time series images is shown on the right. (c) Time course tracing of lumen diameter ( $n=6)$. (Color online only.)

cium reservoir, can also lead to an increase in the intracellular calcium concentration. In addition, the laser may affect the actions of membrane ion channels important in calcium

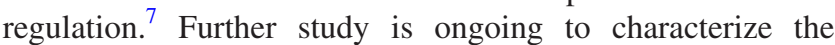
mechanisms of pulsed laser effects on VSMCs and other cell types.

Most of the irradiated cell showed punctate calcium distribution in the cytosol at the later time point. It exhibited membranous morphology and existed only in the cytosol. Because it appeared at the later time point when the calcium signal dropped, the punctate calcium pattern may be related to the calcium reuptake process. ${ }^{17}$ Combining these, the punctate calcium pattern may be endoplasmic recticulum or mitochondria. Especially because mitochondria can have dynamic fusion and fission depending on the change in cellular environment, ${ }^{18}$ further study is ongoing on the effect of laser on mitochondria.

In the animal experiment, laser stimulation induced circular vasoconstriction. Because the calcium wave from the irradiated cell propagates to the neighboring cells in vitro, similar calcium signal propagation might occur in vivo. It is speculated that the calcium signal from the irradiated cell propagates circularly and longitudinally to some extent, resulting in the synchronous vascular constriction.

The control of vascular tone in the brain is mediated by multiple cell types, including endothelial cells, smooth muscle cells, neurons, and astrocytes. ${ }^{19}$ Although we tried to avoid the inner layer and to irradiate the media layer by targeting a region 3 to $5 \mu \mathrm{m}$ from the luminal surface, we must consider that the outer layer of the vessel wall is in contact with astrocytic endfeet and neuronal processes. Therefore, not only smooth muscle cells but also other cell types may be responsible for laser-induced arterial contraction. The initial response to irradiation was uniformly vasoconstriction, with no case of vasodilation, which is often observed with electrical stimulation in neurons ${ }^{19}$ or photolysis of a caged-Ca ${ }^{2+}$ compound in astrocytes ${ }^{20,21}$ in vivo. Furthermore, similar arterial contraction was observed in dermal tissues, which lack neurons and astrocytes, collectively indicating that vascular smooth muscle can contract in vivo with laser stimulation (data not shown). Still, the involvement of astrocytes, neurons, or even endothelial cells in laser-induced cortical arterial contraction cannot be precluded completely, and further study is needed.

\section{Acknowledgments}

This research was supported by a grant (2009K001282) from the Brain Research Center of the 21st Century Frontier Research Program funded by the Ministry of Education, Science, and Technology, Korea. 


\section{References}

1. J. F. Brekke, W. F. Jackson, and S. S. Segal, "Arteriolar smooth muscle $\mathrm{Ca} 2+$ dynamics during blood flow control in hamster cheek pouch," J. Appl. Physiol. 101(1), 307-315 (2006).

2. M. J. Berridge, "Smooth muscle cell calcium activation mechanisms," J. Physiol. (London) 586(Pt 21), 5047-5061 (2008)

3. C. Iadecola, "Neurovascular regulation in the normal brain and in Alzheimer's disease," Nat. Rev. Neurosci. 5(5), 347-360 (2004).

4. H. Girouard and C. Iadecola, "Neurovascular coupling in the normal brain and in hypertension, stroke, and Alzheimer disease," J. Appl. Physiol. 100(1), 328-335 (2006).

5. R. Aras-Lopez, J. Blanco-Rivero, F. E. Xavier, M. Salaices, M. Ferrer, and G. Balfagon, "Dexamethasone decreases contraction to electrical field stimulation in mesenteric arteries from spontaneously hypertensive rats through decreases in thromboxane A2 release," $J$ Pharmacol. Exp. Ther. 322(3), 1129-1136 (2007).

6. J. Ando, N. I. Smith, K. Fujita, and S. Kawata, "Photogeneration of membrane potential hyperpolarization and depolarization in nonexcitable cells," Eur. Biophys. J. 38(2), 255-262 (2009).

7. N. I. Smith, K. Fujita, T. Kaneko, K. Katoh, O. Nakamura, S. Kawata, and T. Takamatsu, "Generation of calcium waves in living cells by pulsed-laser-induced photodisruption," Appl. Phys. Lett. 79(8), 1208-1210 (2001).

8. W. Watanabe and N. Arakawa, "Femtosecond laser disruption of subcellular organelles in a living cell," Opt. Express 12(18), 4203-4213 (2004).

9. U. K. Tirlapur and K. Konig, "Targeted transfection by femtosecond laser," Nature 418(6895), 290-291 (2002).

10. H. Hirase, V. Nikolenko, J. H. Goldberg, and R. Yuste, "Multiphoton stimulation of neurons," J. Neurobiol. 51(3), 237-247 (2002).

11. N. Nishimura, C. B. Schaffer, B. Friedman, P. S. Tsai, P. D. Lyden, and D. Kleinfeld, "Targeted insult to subsurface cortical blood vessels using ultrashort laser pulses: three models of stroke," Nat. Methods 3(2), 99-108 (2006).
12. N. I. Smith, Y. Kumamoto, S. Iwanaga, J. Ando, K. Fujita, and S. Kawata, "A femtosecond laser pacemaker for heart muscle cells," Opt. Express 16(12), 8604-8616 (2008).

13. R. K. Dubey, D. G. Gillespie, K. Osaka, F. Suzuki, and E. K. Jackson, "Adenosine inhibits growth of rat aortic smooth muscle cells. Possible role of A2b receptor," Hypertension 27(3 Pt 2), 786-793 (1996).

14. H. T. Xu, F. Pan, G. Yang, and W. B. Gan, "Choice of cranial window type for in vivo imaging affects dendritic spine turnover in the cortex," Nat. Neurosci. 10(5), 549-551 (2007).

15. H. Niioka, N. I. Smith, K. Fujita, Y. Inouye, and S. Kawata, "Femtosecond laser nano-ablation in fixed and non-fixed cultured cells," Opt. Express 16(19), 14476-14495 (2008).

16. L. Paterson, B. Agate, M. Comrie, R. Ferguson, T. Lake, J. Morris, A. Carruthers, C. T. Brown, W. Sibbett, P. Bryant, F. Gunn-Moore, A. Riches, and K. Dholakia, "Photoporation and cell transfection using a violet diode laser," Opt. Express 13(2), 595-600 (2005).

17. R. T. Dirksen, "Sarcoplasmic reticulum-mitochondrial through-space coupling in skeletal muscle," Appl. Physiol. Nutr. Metab. 34(3), 389_ 395 (2009)

18. D. G. Breckenridge, M. Stojanovic, R. C. Marcellus, and G. C. Shore, "Caspase cleavage product of BAP31 induces mitochondrial fission through endoplasmic reticulum calcium signals, enhancing cytochrome c release to the cytosol," J. Cell Biol. 160(7), 1115-1127 (2003).

19. P. G. Haydon and G. Carmignoto, "Astrocyte control of synaptic transmission and neurovascular coupling," Physiol. Rev. 86(3), 1009_ 1031 (2006).

20. T. Takano, G. F. Tian, W. Peng, N. Lou, W. Libionka, X. Han, and M. Nedergaard, "Astrocyte-mediated control of cerebral blood flow," Nat. Neurosci. 9(2), 260-267 (2006).

21. M. R. Metea and E. A. Newman, "Glial cells dilate and constrict blood vessels: a mechanism of neurovascular coupling," J. Neurosci. 26(11), 2862-2870 (2006). 